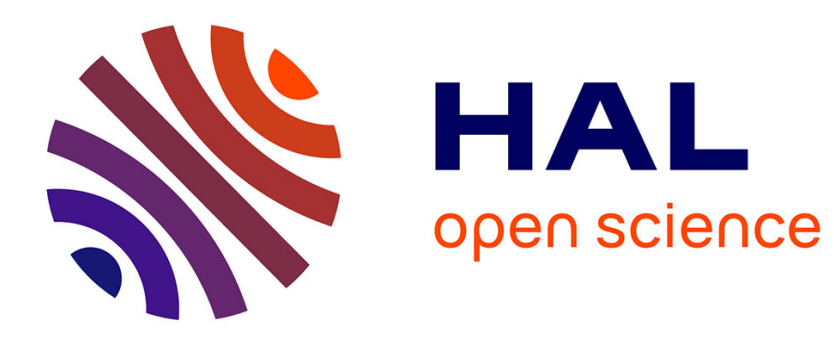

\title{
Le point de vue de l'agent de terrain
}

André Moulin

\section{To cite this version:}

André Moulin. Le point de vue de l'agent de terrain. Revue forestière française, 1972, 24 (S), pp.619622. $10.4267 / 2042 / 20676$. hal- 03395645

\section{HAL Id: hal-03395645 \\ https://hal.science/hal-03395645}

Submitted on 22 Oct 2021

HAL is a multi-disciplinary open access archive for the deposit and dissemination of scientific research documents, whether they are published or not. The documents may come from teaching and research institutions in France or abroad, or from public or private research centers.
L'archive ouverte pluridisciplinaire HAL, est destinée au dépôt et à la diffusion de documents scientifiques de niveau recherche, publiés ou non, émanant des établissements d'enseignement et de recherche français ou étrangers, des laboratoires publics ou privés. 
En outre, une importante propagande était menée auprès des collectivités locales qui détiennent en Lozère 70000 hectares de biens sectionaux dont 20000 seulement étaient soumis au Régime forestier. Les autres, anciennement loués aux troupeaux transhumants sous la forme de "nuits de fumature " étaient soit sous-exploités soit totalement abandonnés avec retour à la lande à genêts puis à callune.

Peu à peu, des milliers d'hectares firent l'objet d'aménagements sylvo-pastoraux. Notamment, près de 7000 hectares de terrains ont été aménagés au titre des actions d'intérêt mixte pastoral et forestier.

En guise de conclusion, nous dirons que trois leçons peuvent se dégager au vu des actions menées depuis 25 ans en Lozère gråce aux aides du Fonds forestier national :

- une leçon d'humilité car, si 33000 hectares ont pa être reboisés à ce jour, il en reste encore 100000 d mettre en valeur par la forêt sans nuire en aucune manière aux activités agricoles et pastorales. D'autre part, pour quelques progrès accomplis sur le plan technique, combien en reste-t-il encore à faire dans tous les domaines?

-une leçon de persévérance car d'autres que nous pourront seuls juger des efforts d'aujourd'hui : il faut beaucoup de foi pour poursuivre auprès des collectivités locales ou des particuliers ce patient travail de restructuration foncière, d'aménagement rural au bénéfice des générations futures.

- une leçon d'espoir enfin: ces jeunes plantations croissent avec vigueur à l'ombre des vieilles futaies créées par les anciens. L'exemple du passé est un gage certain de leur réussite dans l'avenir.

\section{le point de vue de l'agent de terrain}

La promotion d'une politique forestière particulièrement active tendant notamment à la mise en valeur de terrains à vocation forestière, à la reconstitution de peuplements surexploités ou détruits ainsi qu'à une meilleure utilisation d'une production ligneuse à améliorer selon l'évolution des débouchés commerciaux et industriels, ne pouvait être mise à la charge du personnel traditionnel de l'Administration forestière.

II convenait donc, à la création du Fonds forestier national, de procéder au recrutement et à la mise en place d'un personnel technique et administratif, réparti entre les services de la Direction générale et ceux des conservations départementales en fonction des besoins forestiers locaux.

Le corps technique fut constitué d'ingénieurs, sous-ingénieurs, agents de maîtrise et gardes. Le cadre administratif comprenait, quant à lui, des secrétaires administratifs principaux et des secrétaires administratifs. 
Les moyens d'intervention du Fonds forestier national consistant alors en l'attribution de subventions, de prêts en numéraires et prêts en travaux (contrats) étaient définis dans un ensemble de textes précis.

Tout semblait alors facile, mais dès les débuts, le personnel dut principalement faire œuvre de persuasion pour que soit acceptée cette nouvelle orientation de la politique forestière d'une administration ancienne mais néanmoins peu connue surtout des propriétaires privés.

Cette période de démarrage fut relativement courte puisque dés 1950-1951 la mise en œuvre du Fonds forestier national entrait dans une phase active.

Mais peut-être vaut-il mieux laisser la parole à certains " praticiens \#, technique et administratif, du Fonds forestier qui participent depuis l'origine a la promotion et a l'intensification des activités de cet élément important de la politique forestiére :

"Figurant au nombre des plus anciens du cadre technique, puisque recruté à sa création, je crois pouvoir dire que notre action et nos fonctions sur le terrain notamment en forêt privée ont évolué et évoluent encore très rapidement selon une courbe ascendante parallèle à celle qui matérialiserait la multiplicité des interventions du Fonds forestier.

Au cours des années 1948 et 1949 notre action fut surtout axée sur la diffusion des modalités d'aides consenties au bénéfice des propriétaires de bois et de terrains á vocation forestlère.

A cette époque-lá, nous ne disposions que de trois moyens d'intervention : les subventions, les prêts en numéraires et les prêts en travaux.

Dés les premiers contacts, il fallut se rendre à l'évidence : la subvention était la forme la mieux adaptée à la petite propriété, dont les détenteurs se trouvent généralement en milieu rural.

Elle s'avéra être le meilleur outil de propagande et connut rapidement un assez grand succès malgré certaines réticences résultant de l'appréhension d'un risque de gestion autoritaire par l'État ou du risque d'une réquisition facilitée en cas de crise ou de guerre ou encore de l'éventuelle ingérence de l'État lors des exploitations forestiéres

Le prêt travaux (contrat) recueillit assez vite en raison même des avantages exceptionnels de cette formule l'accord des propriétaires forestiers plus importants.

Dès 1950 nous entrions dans une phase active puisque un certain nombre d'opérations par subventions, prêts-travaux et prêts en numéraires se réalisaient.

Considérant alars que ces créations constituaient la meilleure propagande, notre action revêtit plusieurs formes, l'information des possibilités offertes, la dissuasion des réticences et l'organisation de contacts entre propriétaires ayant déjá bénéficié des aides du F.F.N. et ceux ayant l'intention de les solliciter. Ces contacts étaient bien entendu assortis de visites des opérations réalisées et de nombreux commentaires d'autant plus faciles à fournir que nous avions pratiquement élaboré nous-mêmes les projets et dirigé les travaux.

L'élan était donné mais il fallait susciter des vocations forestiêres chez les propriétaires. De nombreuses réunions furent alors organisées dans les mairies, ainsi que dans les Chambres de Commerce ; de même des conférences furent faites â la radio, au cours d'émissions agricoles.

Notre rôle alors n'était plus seulement d'informer des possibilités ou de réduire les quelques réticences rencontrées, il fallait démontrer d'une manière exhaustive la rentabilité des opérations. l'intérêt économique général et particulier, assurer les propriétaires de l'assistance de notre service.

Dès lors, les demandes se firent nombreuses, émanant de toutes les classes sociales. Notre argumentation devint évidemment plus nuancée selon la situation de chaque requérant.

Mais parallélement à ces besoins de boisement ou reboisement et d'équipement de la forêt, d'autres nécessités, dans le même esprit d'amélioration de la potentialité de la forêt et de meilleure utilisation de la production ligneuse s'exprimerent : il fallait éviter les risques de démembrement à l'occasion des successions et il était également urgent de favoriser la création de grands ensembles forestiers par associations de petits propriétaires.

En outre, le regroupement et la modernisation des scieries d'une part et des pépiniéres privées d'autre part furent aussi pris en considération par le Fonds forestier qui arrêta de nouvelles dispositions d'assistance technique et financière. 
Afin d'améliorer la qualité des peuplements à créer, un contrôle des pépiniéristes privés fut créé en même temps que la normalisation de certaines essences forestières et la sélection des graines. L'application de l'ensemble de ces dispositions fit partie de nos attributions.

Mais je ne voudrais pas faire ici l'énumération chronologique, et par là-même fastidieuse, des divers modes biens connus maintenant d'intervention du Fonds forestier national et parallèlement des différents aspects de notre róle et de notre action d'informateur, de conseiller technique, de directeur des travaux et de gestionnaire des réalisations.

Je me contenterai de dire que chaque nouvelle modalité d'aide nous met en contact avec un public sans cesse renouvelé, de tous horizons sociaux, d'intérêts extrêmement divers et qu'il est donc absolument nécessaire que nous soyions, en même temps que des praticiens possédant bien les nouvelles techniques sans cesse en évolution, des psychologues avertis connaissant d'une façon approfondie les besoins et l'état d'esprit de chaque candidat, même en puissance, à l'aide du Fonds forestier.

Tels furent au sein des Conservations des Eaux et Forêts notre rôle et nos fonctions jusqu'en 1965, époque de la réforme du Ministère de l'Agriculture qui se traduisit par la création de l'Office national des forêts d'une part et, d'autre part, des Directions départementales de l'agriculture dont l'effectif forestier fut constitué en grande partie par le personnel F.F.N.

En principe nos attributions restaient les mêmes. En fait et par suite de certaines situations administratives, nous nous sommes trouvés dans bien des cas avoir la responsabilité totale du service F.F.N., puis de l'ensemble des questions spécifiquement forestières, restauration des terrains en montagne exclue, et devenir ainsi les seuls collaborateurs forestiers des directeurs de l'Agriculture représentant l'autorité de tutelle de l'Office national des forêts.

De ce fait, nombreux parmi nous sont en qualité de mandataires de leurs directeurs,en relation permanente et directe soit avec les ingénieurs en chef du Génie rural, des Eaux et des Forêts, chefs des services régionaux d'aménagement forestier, soit avec les ingénieurs du Génie rural des Eaux et des Forêts, chefs de Centres de gestion de l'O.N.F., avec lesquels ils sont appelés à prendre des initiatives ou des positions parfois délicates. II va sans dire que notre rôle est difficile et qu'une parfaite connaissance des interlocuteurs facilite souvent bien des rapports dans l'intérêt même du Service, notamment à l'occasion de l'établissement des programmes annuels d'investissements forestiers ou de modifications à apporter, en cours d'exécution de travaux dans le cadre des contrats ou primes aux techniques prévues initialement.

Et puis, toujours parce que le personnel du Fonds forestier est souvent le seul élement forestier des Directions départementales de l'agriculture, nous sommes appelés à connaitre effectivement d'autres problèmes tels que ceux posés par les défrichements, l'application de la loi Sérot-amendement Monichon, la protection de la nature, la création de forêts récréatives.

Je ne citerai, en outre, que pour mémoire, la représentation assez fréquente de nos directeurs départementaux au sein de réunions diverses, séances d'adjudication, réception de travaux en forêts soumises, Chambres d'Agriculture, Préfecture, etc.

Ceci, sans parler de quelques camarades chargés de gestion de pépiniéres administratives et vergers à graines.

Je crois avoir brossé là un tableau reflétant de façon objective le rôle, l'action et les fonctions du cadre technique F.F.N. qui doit par ailleurs se tenir au courant des modifications fréquentes des textes codifiant les différentes actions du Fonds. Il est vrai qu'en cette matière nous faisons toujours appel à nos camarades administratifs sans lesquels d'ailleurs l'efficacité de notre activité serait très fortement réduite.

Mais je profite de la présence de l'un d'eux et lui cède la parole.

"L'expérience que j'ai des textes régissant les diverses modalités d'intervention du F.F.N. résulte de mon ancienneté dans le cadre administratif auquel j'appartiens depuis sa création, il y a 25 ans.

De même que nos collégues du cadre technique, et pour les mêmes raisons, notre évolution a été très rapide. Toutefois nous avons do, non seulement nous imprégner littéralement des dispositions administratives dont la complexité inévitable au fur et à mesure de la mise en ceuvre rendait l'application délicate ou difficile, mais aussi nous tenir informés de l'évolution des techniques pour remplir avec efficacité notre róle d'informateur et surtout de conseiller. 
En effet, nos fonctions d'administratif nous conférent une présence continuelle au bureau qui donne aux propriétaires désireux de renseignements une assurance de toujours trouver un interlocuteur qu'ils veulent compétent aussi bien sur les problemes administratifs que sur les aspects techniques et économiques de l'ensemble des questions forestierres F.F.N. et autres.

Lors de la réforme administrative intervenue en 1965, nous avons également été rattachés d l'Administration d'Etat au sein des Directions départementales de l'agriculture où notre action a pris une importance accrue sur les plans administratif et technique en raison des situations nouvelles ainsi créées. Certains d'entre nous se trouvent d'ailleurs investis des attributions des ex-Conservateurs des Eaux et foréts.

Nos contacts avec les Services régionaux d'aménagement forestier sont fréquents de même qu'avec les Chambres d'agriculture, les Préfectures et les différents services de l'Office national des forêts qui font appel au cadre administratif en tant que spécialiste des questions F.F.N.

Nous sommes également, comme nos camarades du cadre technique, chargés de la représentation de nos Directeurs au sein de réunions diverses à l'échelon départemental, voire régional. "

Voici donc exposés succinctement le rôle et les fonctions de chacun des deux cadres du Fonds forestier national. Les résultats obtenus en 25 années, dont deux de démarrage, sont considérables et le personnel F.F.N. technique et administratif y a pris une part des plus actives dans toutes les disciplines. L'effort soutenu pendant ce quart de siècle doit être un encouragement pour mener à bonne fin encore de nombreuses réalisations dans l'optique d'un meilleur service de la forêt et de la Nation.

II ne semble donc pas que la pérennité du Fonds forestier national puisse maintenant être remise en cause mais blen au contraire il apparaît éminemment souhaitable de le considérer comme un département du Ministère de l'Agriculture et du développement rural doté d'un personnel ayant la qualité de fonctionnaire à part entière.

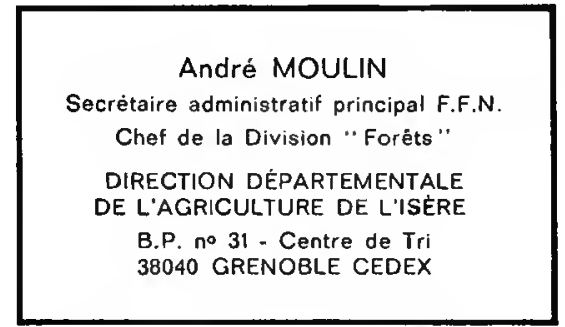

